\title{
The NHS Bowel Cancer Screening Program: current perspectives on strategies for improvement
}

This article was published in the following Dove Press journal: Risk Management and Healthcare Policy

\author{
Sara Koo' \\ Laura Jane Neilson' \\ Christian Von Wagner ${ }^{2}$ \\ Colin John Rees ${ }^{1,3,4}$ \\ 'Department of Gastroenterology, \\ South Tyneside District Hospital, \\ South Shields, ${ }^{2}$ Health Behaviour \\ Research Centre, University \\ College London, London, ${ }^{3} \mathrm{~S} c h o o l$ \\ of Medicine, Pharmacy and Health, \\ Durham University, Stockton on \\ Tees, ${ }^{4}$ Northern Institute for Cancer \\ Research, Newcastle University, \\ Newcastle upon Tyne, UK
}

\begin{abstract}
Colorectal cancer (CRC) is the third most common cancer in the UK. The English National Health Service (NHS) Bowel Cancer Screening Program (BCSP) was introduced in 2006 to improve CRC mortality by earlier detection of CRC. It is now offered to patients aged 60-74 years and involves a home-based guaiac fecal occult blood test (gFOBt) biennially, and if positive, patients are offered a colonoscopy. This has been associated with a $15 \%$ reduction in mortality. In 2013, an additional arm to BCSP was introduced, Bowelscope. This offers patients aged 55 years a one-off flexible sigmoidoscopy, and if several adenomas are found, the patients are offered a completion colonoscopy. BCSP has been associated with a significant stage shift in CRC diagnosis; however, the uptake of bowel cancer screening remains lower than that for other screening programs. Further work is required to understand the reasons for nonparticipation of patients to ensure optimal uptake. A change of gFOBt kit to the fecal immunochemical tests (FIT) in the English BCSP may further increase patient participation. This, in addition to increased yield of neoplasia and cancers with the FIT kit, is likely to further improve CRC outcomes in the screened population.
\end{abstract}

Keywords: colorectal cancer, flexible sigmoidoscopy screening, Bowelscope, CT colongraphy, fecal immunochemical tests, gFOBt screening, uptake, quality in colonoscopy

\section{Introduction}

Colorectal cancer $(\mathrm{CRC})$ is the third most common cancer diagnosis in the UK. ${ }^{1}$ Its incidence has risen by $5 \%$ in the past decade, and it is the second most common cancer death in the UK with a 5 -year survival of $59 \%{ }^{1}$

The English National Health Service (NHS) Bowel Cancer Screening Program (BCSP) was introduced in 2006 to improve the outcomes and mortality of CRC by its earlier detection and thereby prevention of CRC. The program achieved full roll out in England and Wales in 2010. Eligible patients are invited to complete a homebased guaiac fecal occult blood test ( $\mathrm{gFOBt}$ ) biennially. The test looks for tiny traces of blood. Typically, occult blood is detected in $2 \%$ of those who participate, and they are then followed up with a diagnostic test (most commonly colonoscopy). ${ }^{2}$ The NHS BCSP was initially targeted at those aged 60-69 years but the upper age limit was extended to 74 years in 2010. In the past 10 years, over 30 million people have been invited to participate in the BCSP. ${ }^{3}$ In addition, "Bowelscope" screening was introduced in England in 2013. This involves a one-off flexible sigmoidoscopy at age 55 years, with adenoma removal and completion colonoscopy for those with significant findings at flexible sigmoidoscopy. CRC screening in Scotland differs, in that biennial
Correspondence: Colin J Rees

Department of Gastroenterology, South Tyneside District Hospital, Harton Lane, South Shields NE34 OPL, UK

Email Colin.Rees@stft.nhs.uk 
screening is offered to those aged 50-74 years. At present, there is no Bowelscope screening in Scotland. In Wales and Northern Ireland, screening is similar to that in England in that gFOBt screening is offered to individuals aged 60-74 years; however, there is currently no Bowelscope screening arm of the program.

Organized CRC screening has been shown to detect $\mathrm{CRC}$ at an earlier stage, thereby improving morbidity and mortality. ${ }^{2}$ The initial UK screening trial conducted in Nottingham offered biennial gFOBt, with colonoscopy where positive, to patients aged 45-74 years and demonstrated a $15 \%$ reduction in CRC mortality. ${ }^{4}$ Colonoscopy screening also gives the opportunity to detect adenomas, some of which are precursors to CRC, meaning that adenomas maybe removed thereby preventing development of CRC. ${ }^{5}$ This paper reviews the NHS BCSP and discusses potential future direction.

\section{Guaiac-based FOBt screening process}

gFOBt population-based screening has been shown to improve CRC outcomes by reducing CRC mortality. Trials were conducted in Nottingham, UK; Funen, Denmark, and Minnesota, USA., ${ }^{4,67}$ The UK trial ran from 1985 and involved biennial screening utilizing non-rehydrated gFOBt in patients aged 50-74 years. ${ }^{4}$ Patients were followed up for a median of 7.8 years (4.5-14.5 years), and individuals in the screening arm were offered gFOBt screening between 3 and 6 times. ${ }^{4}$ In this study, $2.1 \%$ of gFOBt were positive, and its positive predictive values (PPVs) of neoplasia and cancer were $47.1 \%$ and $9.9 \%$, respectively. ${ }^{4}$ Most importantly, the study demonstrated a $15 \%$ reduction in CRC mortality through biennial gFOBT screening. ${ }^{4}$ The Danish trial methodology was similar to biennial screening, involving non-rehydrated gFOBt with 10-year follow-up. ${ }^{6}$ Its main difference to the Nottingham study was that only responders during the initial round of screening were invited for subsequent rounds of screening. This study revealed a CRC reduction of $18 \%{ }^{6}$ The UK and Danish trials both revealed that nonresponders who were subsequently found to have CRC were commonly diagnosed with Dukes B, C, or D tumors. In the screened population, a stage shift was demonstrated, meaning that screen-detected cancers were more likely to be at an early stage (Dukes A or B). ${ }^{4,6}$ Furthermore, the mortality rate of screen-detected cancers was lower than that in the control group during follow-up. ${ }^{4,6}$ In contrast to these studies, the American trial used mostly rehydrated gFOBt testing, comparing annual and biennial screening and a control group (no screening). ${ }^{7}$
Patients were followed up for 13 years. Annual screening was associated with $33 \%$ reduction in CRC mortality, whereas biennial screening reduced mortality by $6 \%$ (nonsignificant). ${ }^{7}$ The reason for the disparity between annual screening and biennial screening is unclear.

The BCSP offers gFOBt screening to those aged 60 and 74 years biennially. An invitation letter and information leaflet on bowel cancer and the BCSP are posted to patients, followed by a gFOBt kit. The kit contains six windows, in which patients are asked to fill with two fecal samples from three separate bowel motions. No dietary restrictions are required during the sampling period. Patients are asked to return the completed kit via freepost within a 14-day period from the initial bowel motion with a view to receiving a result letter within 2 weeks. Currently, in the UK, the gFOBt test kit is a non-rehydrated guaiac-based kit (Hema-screen ${ }^{\mathrm{TM}}$ ). The test card is coated with guaiac, a reagent. Haem complexes contain pseudoperixodase, and when blood is present in stool, pseudoperoxidase in haem reacts with guaiac. When analyzed with hydrogen peroxide, this turns the sample blue.

Early data from the NHS BCSP in 2013 demonstrated a gFOBt positivity rate of $2.08 \%$, suggesting that a large proportion of gFOBt tests are normal (no positive windows). ${ }^{8}$ Where 1-4 windows are positive, this is regarded as a "weak positive," and patients will require two completely negative repeat tests before discharge from that round of screening. However, if the repeat tests have any positive windows, patients proceed to a specialist screening practitioner (SSP) review to discuss colonoscopy. An abnormal gFOBt is regarded as $\geq 5$ positive windows (out of 6 ). These patients proceed to review by SSP to discuss colonoscopy. In England, gFOBt kits are used throughout each stage of screening (until 2018). However, in Scotland, Wales, and Northern Ireland, when a repeat test is required for a "weak positive" test, fecal immunochemical tests (FIT) kits are used. The two-tiered system involves retesting all positive gFOBt samples with the FIT kit. This is to prevent false-positive cases as the FIT kit is capable of detecting lower thresholds of blood present in stools. The differences between each kit are discussed in detail in "The future: FIT versus FOB" section.

Within the English BCSP, patients who undergo polypectomy at colonoscopy are offered an SSP clinic appointment to discuss results and are offered ongoing adenoma surveillance based on the British Society of Gastroenterology (BSG) guidelines (Figure 1). ${ }^{9}$ Those diagnosed with CRC are referred to the local hospital multidisciplinary meeting for further investigation and ongoing treatment. Patients with a normal colonoscopy or low-risk polyps are discharged and 


\section{SURVEILLANCE FOLLOWING ADENOMA REMOVAL}



Figure I British Society of Gastroenterology guidelines for adenoma surveillance.

Note: Reproduced from Guidelines for colorectal cancer screening and surveillance in moderate and high risk groups (update from 2002), Cairns et al, 59, 666-690, 2010 with permission from BMJ Publishing Group Ltd. ${ }^{9}$

reinvited for further gFOBt testing as per BCSP. Patients with intermediate risk (at least one adenoma $\geq 1 \mathrm{~cm}$ in size or 3-4 small adenomas) or high-risk adenomas (more than three adenomas with one $\geq 1 \mathrm{~cm}$ in size or five or more small adenomas) at baseline colonoscopy are invited for a further colonoscopy for adenoma surveillance - high risk at 12 months and intermediate risk at 36 months. ${ }^{9}$ Patients on the adenoma surveillance programs are excluded from subsequent gFOBt rounds until adenoma surveillance is complete. Patients with intermediate risk adenomas at baseline colonoscopy require two normal consecutive colonoscopies prior to discharge from the surveillance program, whereas those with high-risk adenomas require three consecutive normal colonoscopies. ${ }^{9}$

Between 2009 and 2011, 90.6\% of colonoscopies were performed following a "weak positive" gFOBt and $9.4 \%$ following an abnormal result. ${ }^{10} \mathrm{O}$ all colonoscopies performed, $29.8 \%$ were normal and noncancerous findings were common (11.5\%); most commonly, diverticular disease (33\%), ulcerative colitis (15\%), angiodysplasia (4.5\%), Crohn's disease $(3.2 \%)$, rectal ulcer $(2 \%)$, and radiation proctitis $(1.8 \%) .{ }^{2}$ Within the first million returned gFOBt kits (2006 to late 2008), CRC was identified in $10.1 \%$ of those with abnormal and weakly positive gFOBt and high-risk adenomas were identified in $9.8 \%{ }^{2}$ The PPVs for cancer and neoplasia of an abnormal gFOBt are $26 \%$ and $64.9 \%$, respectively. ${ }^{11}$ The risk of CRC was higher in those with abnormal gFOBt (21.3\%-26\%) compared to those with a "weak positive" result $(5.9 \%-9 \%){ }^{10,11}$ Similarly, high-risk adenomas were more commonly seen in those with abnormal gFOBt (16\%) compared to those with a "weak positive" result $(10 \%) .{ }^{11}$ The risk of CRC in a weakly positive gFOBt rose by $2.5 \%$ for every $10 \%$ rise in spot positivity (number of positive windows in each kit for one round of screening). ${ }^{10}$ Geraghty et al retrospectively observed patients diagnosed with cancer after two consecutive rounds of screening, finding that $90.6 \%$ had a normal gFOBt during the preceding round. ${ }^{10}$ It was also noted that patients with a combination of four positive spots in the first test kit but two normal subsequent kits had a similar risk of CRC to those who were offered colonoscopy. ${ }^{10}$ 
Table 1 shows the breakdown of CRC site depending on referral routes. Screen-detected CRCs were most commonly left-sided (74.2\%), with $30 \%$ being rectal cancers, $6.3 \%$ in rectosigmoid, $31.7 \%$ in sigmoid, and $6.3 \%$ in the descending colon. ${ }^{12}$ This contrasts with the pattern of cancer distribution in the symptomatic population. Patients presenting through emergency admissions have more cecal cancers $(34.4 \%)$ compared to sigmoid cancers $(25.1 \%)$ or rectal cancers $(14.0 \%) .{ }^{12}$ On the other hand, patients presenting through general practitioners (GPs) are more likely to have rectal cancers $(35.4 \%)$, followed by cecal cancers $(24.2 \%)$ and sigmoid cancers $(21.8 \%) .{ }^{12}$ Overall screen-detected CRCs are more common in men $(11.6 \%)$ than women $(7.8 \%){ }^{8}$ Rectal cancer is more common in men, whereas right-sided cancers are more common in women. ${ }^{2}$

The majority of bowel cancer cases present via GP referrals $(55 \%)$, followed by emergency presentations $(20 \%)$ and bowel cancer screening (10\%). ${ }^{12}$ Screen-detected cancers are often at an earlier stage compared to those diagnosed via GP referrals or presenting via emergency admissions. ${ }^{12}$ The latter groups were more likely to have advanced disease (nodal involvement or metastatic disease at presentation), with patients presenting via emergency admissions having more advanced disease compared to those referred in from GPs. ${ }^{12}$

The first million returned FOBt kits suggest that $71.3 \%$ of cancers are at an early stage (polyp cancer 9.8\%, Dukes A $32 \%$, Dukes B 29.5\%). ${ }^{2}$ The remaining stages (Dukes $\mathrm{C}$ and D) were found in $25.7 \%$ and $3 \%$ of patients, respectively. ${ }^{2}$ Comparing stage-matched screen-detected cancers to interval cancers (CRC detected in between rounds of screening, after a normal gFOBt or colonoscopy) and cancers identified in screening nonparticipants (patients who did not participate in gFOBt screening), the stage of cancer diagnosis favors patients in screening compared to the other groups. Dukes A cancers were found in $38.8 \%$ patients undergoing screening in

Table I Breakdown of CRC site based on referral route

\begin{tabular}{|c|c|c|c|}
\hline $\begin{array}{l}\text { Site/referral } \\
\text { route }\end{array}$ & Screening (\%) & $\begin{array}{l}\text { Emergency } \\
\text { admission (\%) }\end{array}$ & $\begin{array}{l}\text { GP } \\
\text { referral (\%) }\end{array}$ \\
\hline Rectal & 30 & 14.0 & 35.4 \\
\hline Rectosigmoid & 6.3 & 4.2 & 5.5 \\
\hline Sigmoid colon & 31.7 & 25.1 & 21.8 \\
\hline $\begin{array}{l}\text { Splenic flexure/ } \\
\text { descending colon }\end{array}$ & 6.2 & 9.4 & 4.7 \\
\hline Transverse colon & 5.7 & 8.2 & 5.0 \\
\hline Hepatic flexure & 3.4 & 4.8 & 3.4 \\
\hline $\begin{array}{l}\text { Cecum/ascending } \\
\text { colon }\end{array}$ & 16.9 & 34.4 & 24.2 \\
\hline
\end{tabular}

Note: Data from Braun et al. ${ }^{12}$

Abbreviations: CRC, colorectal cancer; GP, general practitioner. comparison with $13.8 \%-18.8 \%$ in screening nonparticipants and interval cancers. ${ }^{13}$ Conversely, Dukes D cancers were far less common in screen-detected cancers (6.5\%) compared to the latter groups $(22.9 \%-23.2 \%) .{ }^{13}$ More importantly, patients with screen-detected cancers had better outcomes, with a superior 1-year survival (95.9\%) compared to interval cancers or screening nonparticipants $(76.8 \%-78.4 \%) .{ }^{14}$ Additionally, screen-detected Dukes C and D CRC patients had significantly better outcomes compared to interval cancers. ${ }^{13}$ Survival rates in those diagnosed out with the BCSP, including nonparticipants and interval cancers, were not significantly different between routes of diagnosis. ${ }^{15}$

Treatments received also varied among route of cancer diagnosis. Patients with screen-detected cancers were significantly more likely to have curative treatment (88\%) compared to those presenting via GPs $(69 \%)$ or emergency admissions $(52 \%) .{ }^{12}$ They were also more likely to receive major resections $(79.3 \%$ vs $54.4 \%-62.7 \%)$ or local excisions $(9.1 \%$ vs $0.8 \%-3.5 \%$ ) compared to the other routes of diagnosis. ${ }^{12} \mathrm{Of}$ note, non-screen-detected cancers were more likely to not receive any treatment for bowel cancer compared to screendetected cancers $(1.4 \%-4.9 \%$ vs $8 \%){ }^{12}$

\section{Flexible sigmoidoscopy screening}

The adenoma-carcinoma pathway is the main pathway by which some adenomas transform into CRC over $~ 5-10$ years. ${ }^{16}$ Two thirds of colorectal neoplasia are detected in the sigmoid colon and rectum, and distal adenomas plateau at the age of 60 years. ${ }^{17}$ Adenomas over $1 \mathrm{~cm}$ and villous adenomas have higher malignant potential.

The UK Flexible Sigmoidoscopy Screening Trial (UKFSST) included over 170,000 participants aged 55-64 years across 14 centers, who were randomized into the control group or flexible sigmoidoscopy with adenoma clearance. Initially patients were followed up for a median of 11.2 years but have since been extended to 17 years. ${ }^{5}$ The initial CRC incidence and mortality reduction seen were sustained at 17 years follow-up. ${ }^{5}$ In the intention-to-treat analysis (analysis based on all patients randomized to screening arm irrespective of attendance to screening), the incidence of all site CRC and distal CRC was reduced by $26 \%$ and $41 \%$, respectively. ${ }^{5}$ In contrast, for per protocol analysis (analysis based on patients in screening arm who attended for flexible sigmoidoscopy), the reduction of $\mathrm{CRC}$ incidence was greater, with all-site CRC and distal CRC incidence reduced by $35 \%$ and $56 \%$, respectively. ${ }^{5}$ There was no significant effect on proximal $\mathrm{CRC}$ incidence in either the intention-to-treat analysis or per protocol analysis. ${ }^{5}$ Mortality reduction occurred in 30\% for 
all-site CRC, and $46 \%$ for distal CRC in the intention-totreat analysis, but was $41 \%$ and $66 \%$, respectively, in the per protocol analysis. ${ }^{5}$ Proximal CRC mortality reduction was not statistically significant on either analysis. The reduction of CRC incidence and mortality was greater at 17 years followup compared to that at the initial follow-up at the median of 11.2 years.

The findings were similar in the Italian SCORE trial with the same study protocol as the UKFSST trial, with all-site CRC reduction of $18 \%$ (24\% for distal CRC). ${ }^{18}$ Mortality was reduced by $22 \%$, but this was nonsignificant in intention-totreat analyses. ${ }^{18} \mathrm{~A}$ US trial demonstrated reductions in CRC incidence by $21 \%$ and overall mortality by $26 \%{ }^{19}$ In particular, distal cancer incidence was reduced by $29 \%$, associated with a mortality reduction of $50 \% .{ }^{19}$ This trial differed from the UKFSST and SCORE trials in that patients included were aged 55-74 years and received a further screening sigmoidoscopy at either a 3- or 5-year interval. ${ }^{19}$ The US trial found that risk reduction was similar for those aged 55-64 years and 65-74 years. ${ }^{19}$ The NORCCAP study in Norway randomized participants to flexible sigmoidoscopy or flexible sigmoidoscopy in combination with FOBt and revealed no difference in incidence of $\mathrm{CRC}$ at 7 years; however, $\mathrm{CRC}$ incidence was reduced by $20 \%$ and mortality by $27 \%$ after 11 years of follow-up..$^{20}$ The addition of a one-off FOBt did not increase CRC or adenoma detection. ${ }^{20}$ Divergence of mortality between screening and control group occurred after 9 years. ${ }^{20}$

The UKFSST trial found that to prevent one case of CRC, 191 patients needed to be screened at the 11 years follow-up. ${ }^{21}$ This number reduced to 98 at the 17 years follow-up. ${ }^{5}$ The American PLCO trial found that this was higher at $282 .{ }^{19}$

A significant advantage of flexible sigmoidoscopy as a screening investigation is the ability to do the test without full bowel preparation. There are concerns, however, that proximal pathology may be missed, but the UKFSST trial did not reveal a difference in incidence of proximal cancers in the intervention or control group. ${ }^{21}$ The SCORE trial detected a $9 \%$ reduction in proximal CRC, whereas the PLCO trial had a $29 \%$ reduction in proximal CRC with screening. ${ }^{18,19}$

An unexpected finding from the UKFSST trial was that after the first 4 years of expected increase in CRC incidence in the intervention group, incidence in the control group rose steadily compared to the intervention arm. ${ }^{21}$ The SCORE trial findings were similar, but differed in that the crossover was seen at 5 years. ${ }^{18}$ This reaffirms the use of Bowelscope screening in addition to gFOBt testing in maximizing potential of CRC incidence and mortality reduction.

Flexible sigmoidoscopy screening has improved mortality for each stage of cancer, more so for more advanced stages of disease. ${ }^{19}$ The American trial demonstrated that for distal CRC, mortality was reduced by $21.4 \%$ for stage I cancers and by $60.7 \%$ for stage IV cancers. ${ }^{19}$

Bowelscope screening was introduced into the BCSP in 2013, with plans for full roll out in England by 2018. It is a one-off flexible sigmoidoscopy offered to individuals aged 55 years, with an aim to reduce CRC incidence by identifying and removing pre-cancerous adenomas. Individuals aged over 55 years may self-refer for the test up to the age of 60 years, at which point they enter the gFOBt BCSP. Eligible patients are sent an initial invite, with a subsequent appointment invitation and information leaflet. Patients with highrisk features proceed to completion colonoscopy, that is, $\geq 3$ adenomas, single adenoma $\geq 10 \mathrm{~mm}$, tubulovillous adenoma, or $\geq 20$ hyperplastic appearing polyps above the distal rectum.

\section{Uptake}

The benefits of screening programs are maximized with higher uptake. Overall, the uptake of BCSP (gFOBt and Bowelscope) is less than other national screening programs such as breast cancer or cervical cancer where the uptake of these were above $72 \%$ each in 2015/2016..$^{22,23}$ Familiarity and acceptability of the screening program may well play a part.

\section{gFOBt screening}

The initial UK pilot study for gFOBt screening (1985-1991) had an uptake of $56.8 \% .^{24}$ This is similar to the current program uptake of gFOBt screening of $57.9 \%$ between 2012-2015.25 A recent UK study showed that the majority of patients with an abnormal gFOBt attended an SSP clinic (94\%), and $83 \%$ subsequently attended for investigation. ${ }^{8}$ Overall, uptake of colonoscopy following positive gFOBt is $79 \%{ }^{2}$

Data so far suggest that nonresponders of gFOBt screening are more likely to be younger, male, of an ethnically diverse background, or of more deprived socioeconomic status. $^{2}$ Uptake was $4.8 \%$ higher in women compared to men. ${ }^{2}$ Variation of uptake between UK regions also exists, with uptake for all quintiles of deprivation including those who are in the least deprived areas are higher in the North East compared to other regions in the UK. ${ }^{2}$

The unpleasantness associated with obtaining multiple stool samples is a common reason for nonresponding. Other reasons include poor understanding of CRC risk, low health literacy, 
perceptions that screening is not required, that their GP will be consulted if they become symptomatic, or anxiety of possible positive result. ${ }^{26}$ When interviewed on reasons for nonresponding, some individuals had not decided on participation, whereas some had yet to submit the FOB kit due to other priorities. ${ }^{26}$

A face-to-face survey of participants of BCSP screening revealed that only $52 \%$ of survey participants have read almost all of the "Bowel Cancer Screening: The Facts" booklet, whereas $22 \%$ had read none of it. ${ }^{27}$ Furthermore, $31 \%$ of survey participants never participated in BCSP, and of these participants, $63 \%$ never read the booklet. ${ }^{27}$ This suggest that other means of communication with patients who never intended to participate in BCSP is required other than the facts booklet. This may be related to poor health literacy in these individuals. The ASCEND trial assessed four interventions to narrow down the socioeconomic gradient in screening participation, namely, additional supplementary leaflet containing a summary of key information, a narrative of participant's stories, a general practice endorsement of the BCSP, and an enhanced reminder letter. ${ }^{28}$ Sending additional leaflets had no effect on uptake. ${ }^{28}$ Only the enhanced reminder letter that contained a banner with the words "A reminder for you" at the beginning of it followed up with a brief motivational message reduced the socioeconomic gradient in uptake, while GP endorsement significantly increased uptake but did not reduce the socioeconomic gradient. ${ }^{28}$ A recent trial of text message reminders found no significant main effect but a significant $5 \%$ increase in uptake among first-time invitees. ${ }^{29}$

\section{Bowelscope screening}

Uptake of Bowelscope screening is lower than that of gFOBT screening. In the UKFSST, only $53 \%$ of potentially eligible people responded that they would take up the offer of screening when a questionnaire assessing interest in participation was sent. ${ }^{21}$ In the trial, $71 \%$ of those who had been randomized into the intervention group attended screening. ${ }^{21}$ Subsequent feasibility studies of screening reported uptake to be 45\%-55\%. ${ }^{30,31}$ At 14 months after Bowelscope introduction, the uptake in the pilot centers was $43.1 \% .^{32}$

Men were more likely to attend than women (44.6\% vs $41.5 \%$ ), and those in least deprived areas were more likely to participate than those in most deprived areas $(53.2 \%$ vs $32.7 \%) .{ }^{32}$ Appointment slots also had an impact on screening participation. Those who had been offered an appointment out of hours (weekday evenings, Saturday mornings, and afternoons) were more likely to attend than those offered a weekday appointment, but this was nonsignificant in the multivariate analysis. ${ }^{32}$
Individuals may decline to attend screening due to the invasive nature and lack of understanding of flexible sigmoidoscopy. Furthermore, the unfamiliarity of using an enema prior to the test may contribute to this. Other reasons include competing interests, being unable to attend the scheduled appointment, fear of possible endoscopy findings or complications, unwillingness to administer enema, anticipated discomfort, or that participating would waste health care resources. ${ }^{33}$ When screening attenders and nonattenders were interviewed, no interviewees cited the ability of the screening tool to prevent cancer by removing potentially precancerous polyps, suggesting that colorectal polyps are not something that the general public associate with malignancy. ${ }^{33}$

Robb et al evaluated patient response to initial and subsequent reminder invitation in Bowelscope screening. It was found that the proportion of patients responding to the invitation (either accepting, declining, or re-arranging the appointment) were similar $(27 \%-30 \%)$ during the initial or first and second reminder invites. ${ }^{31}$ In another study, when nonresponders were re-invited with a reminder at 12 months, of those who self-referred back for an appointment, $90 \%$ of these individuals (67\% men and 100\% women) requested for same-gender practitioner. ${ }^{34}$ Unlike breast cancer and cervical cancer screening which are gender specific, the practitioner who delivers these screening modalities are more likely to be female. It is not current standard practice in the UK to have same gender practitioner endoscopy lists. Patients may be uncomfortable about the possibility of opposite gender practitioner and decline to participate. This may deter patient participation. For patients in gFOBt screening, this may be addressed during consultation with SSP, but for Bowelscope patients, there is no similar avenue to discuss this. A feasibility study is currently underway evaluating the use of SSPs to increase the uptake in Bowelscope screening. ${ }^{35}$

\section{Quality of endoscopy}

All BCSP colonoscopies are carried out at Joint Advisory Group (JAG)-accredited screening centers by screeningaccredited colonoscopists. Accreditation for BCSP requires a lifetime experience of over 1000 colonoscopies, cecal intubation rate above $90 \%$, and adenoma detection rate (ADR) of over $20 \%$ in the preceding 12 months. All BCSP screeners undergo an accreditation examination and have regular ongoing quality assurance measurements against these benchmark indicators.

BCSP delivers a high standard of colonoscopy. ${ }^{36}$ All colonoscopies performed within the BCSP are recorded in the BCSP database, which has provided a wealth of information 
for research into CRC screening and colonoscopy standards. Through this, several quality indicators have been studied including ADR, polyp detection rate, colonoscopy withdrawal time (CWT), cecal intubation rate, rectal retroversion rate, polyp retrieval rate, sedation practice, bowel preparation scores, and adverse events. ${ }^{37,38}$

The aim of these standards is to reduce variation. Intubation rate of $>90 \%$ is a desired target for colonoscopy, with adequate bowel preparation enabling sufficient adequate diagnostic quality in $\geq 90 \%$ of cases. ADR is used as a surrogate marker for thorough colonic examination. It is widely accepted that improving ADR has a beneficial effect in improving $\mathrm{CRC}$ incidence and mortality, but it has also been associated with reducing interval CRC. ${ }^{39} \mathrm{ADR}$ of $>15 \%$ has been defined as the minimum standard in the symptomatic population; however, ADR in BCSP examinations should exceed $35 \%{ }^{40}$

Mean CWT in the BCSP is 8.4 minutes. ${ }^{41}$ BCSP data suggest that withdrawal time of $>6$ minutes is associated with increased $\mathrm{ADR}$, in particular right-sided lesions. ${ }^{42}$ Lee et al found that the optimal CWT was 10 minutes as this allows for greater attention to deeper folds, difficult corners, and aspiration of pools of fluid. ${ }^{43} \mathrm{CWT}$ above this time did not improve ADR further.

The use of anti-spasmodic therapy such as buscopan has been evaluated in improving ADR. Within the BCSP, buscopan use is associated with a $30 \%$ higher ADR and is routinely recommended where there are no contraindications. ${ }^{42}$ This has not been replicated in meta-analysis of RCTs. ${ }^{44}$ Other areas such as digital rectal examination and rectal retroflexion have also been recommended as they may increase the detection of rectal adenomas.

Good sedation practice is essential to minimize medication-related complications. Table 2 shows the current recommendation for use of pethidine, fentanyl, and midazolam in different age groups from JAG and BSG. ${ }^{45}$ Sedation practice within the BCSP has been shown to be comparable to these recommendations. ${ }^{8}$

Alongside these quality indicators, patient comfort and experience are essential within screening programs and to ensure patient reattendance. In the BCSP, the mean

Table 2 Current recommended sedation practice

\begin{tabular}{lll}
\hline & $<\mathbf{7 0}$ years & $>\mathbf{7 0}$ years \\
\hline Pethidine & $\leq 50 \mathrm{mg}$ & $\leq 25 \mathrm{mg}$ \\
Fentanyl & $\leq 100 \mu \mathrm{g}$ & $\leq 50 \mu \mathrm{g}$ \\
Midazolam & $\leq 5 \mathrm{mg}$ & $\leq 2 \mathrm{mg}$ \\
\hline
\end{tabular}

Note: Data from British Society of Gastroenterology. ${ }^{45}$ colonoscopy procedure time was 26.2 minutes. ${ }^{41}$ Data suggest that most cases in BCSP are well tolerated, with $91.1 \%$ of cases rated as no discomfort or mild or minimal discomfort. ${ }^{46}$ Cases with moderate or severe discomfort (8.9\%) were associated with longer insertion and withdrawal times. ${ }^{46}$ Cases with significant discomfort had similar ADRs to cases where the colonoscopy was well tolerated. There was no association between the use of sedation or analgesia and significant discomfort. $^{46}$

Colonoscopy carries a small risk of adverse events and complications, namely, bleeding and perforation. The risk of these occurring is small in diagnostic procedures and increases with the use of therapy. The most common adverse event reported was bleeding post-polypectomy (42 cases), followed by colonic perforation (12 cases). ${ }^{2}$ The risk of bleeding was found to be at $0.26 \%$ and colonic perforation of $0.04 \%{ }^{37}$

Quality assurance also extends to Bowelscope screening. The benchmark ADR in Bowelscope is $12.1 \%$ based on the UKFSST. ${ }^{47}$ Bowelscope pilot data (2013/2014) revealed an average ADR of $9.2 \% .{ }^{48}$ The difference in ADR between these studies could be related to different age groups recruited (UKFSST - age 55-64 years; Bowelscope - age 55 years only). Furthermore, Bowelscope screening is an open invitation to everyone aged 55 years and reflects real-world data, whereas the UKFSST study population were self-selecting in that they registered interest in participation. Both the studies found significant variation of ADR between endoscopists. This was not accounted for by adjusting confounding factors and was due to difference in performance among endoscopists. ${ }^{47}$ The Bowelscope pilot identified that adenomas were more commonly detected in males $(11.5 \%)$ than females $(6.7 \%)$, and gender-adjusted ADR has been suggested to assess an endoscopist performance. ${ }^{48}$ Further review of this when Bowelscope is fully rolled out would help to evaluate this further.

\section{CT colonography (CTC) compared to colonoscopy}

Patients with abnormal gFOBt or flexible sigmoidoscopy with high-risk features in Bowelscope screening are invited for review with SSP to discuss colonoscopy. Occasionally, patients are deemed unfit for this $(<3 \%) .^{2}$ Most patients underwent a colonoscopy (97\%), followed by CTC (2\%), plain CT abdomen (0.4\%), and barium enema $(0.3 \%){ }^{8}$ Although commonly perceived as less invasive, CTC still requires bowel preparation, although the volume of ingestion is much less. This could either be in the form of picolax or gastrograffin which also has dual function for fecal tagging. 
CTC requires rectal probe insertion for aiding bowel distension with either room air or carbon dioxide. Furthermore, CTC requires movement between prone and supine positions.

In the nonscreening population, $\mathrm{CTC}$ is a reasonable alternative to colonoscopy in patients deemed unfit for colonoscopy. European Society of Gastrointestinal Endoscopy (ESGE) guidance suggests that CTC is recommended over barium enema for the diagnosis of colorectal neoplasia. ${ }^{49}$ A meta-analysis of CTC suggests that it has a sensitivity of $96 \%$ in identifying CRC and polyps in symptomatic and screening patients. ${ }^{50}$ The SIGGAR trial (a multicentered, randomized control trial) evaluated CTC in comparison with colonoscopy in the symptomatic cohort and identified that CTC is comparable to colonoscopy in identification of cancers or polyps above $1 \mathrm{~cm}$ where the pickup rate was $11 \%$ on both the arms. ${ }^{51}$

Complications with CTC are rare $(0.5 \%) .{ }^{52}$ The most significant complication associated with CTC is colonic perforation which may occur during active diverticulitis or acute colitis in patients undergoing CTC. A meta-analysis revealed that CTC-related colonic perforation was low at $0.01 \%{ }^{53}$ Radiation exposure is an often-cited consideration when considering CTC over colonoscopy; however, the radiation risk associated with a single CTC is low. A study reported that the lifetime risk of cancer is increased by $0.2 \%$ when undergoing screening every 5 years between the ages of 50 and 80 years. ${ }^{54}$ Additionally, CTC may also pick up extracolonic findings. These findings may include new diagnosis of non-CRC cancer or other significant noncancer findings such as abdominal aortic aneurysm or adrenal masses. In the SIGGAR trial, in patients randomized to the CTC arm, $30 \%$ of patients were referred for endoscopic evaluation for suspected malignancy, polyps, or uncertainty on imaging, whereas $10 \%$ were referred for the evaluation of extracolonic findings. ${ }^{51}$ Whether utilizing CTC as a first-line investigation is cost-effective due to the need for subsequent investigations from incidental findings which may not be clinically relevant is not yet known.

Although reassuringly safe, the use of CTC in the setting of bowel cancer screening is still being evaluated. Plumb et $\mathrm{al}^{55}$ reviewed CTC as a first-line investigation in patients with subsequent endoscopic investigations through the BCSP database. The authors found that detection of cancers, advanced neoplasia, or large polyps $>1 \mathrm{~cm}$ in size was superior in colonoscopy $(9.0 \%, 32.7 \%, 20.6 \%$, respectively) compared to CTC $\left(4.5 \%, 18.5 \%, 12.4 \%\right.$, respectively). ${ }^{55}$ Of note, this study revealed variation in the detection rates for abnormalities depending on centers, suggesting that experience of radiologists in BCSP may account partly for these findings. Additionally, a meta-analysis in the screening population of patients with a positive FOBt who underwent a CTC found that CTC is $89 \%$ sensitive and $75 \%$ specific for adenomas $\geq 6 \mathrm{~mm} .{ }^{56}$ There is an increasing understanding and research into the use of bowel preparation and fecal tagging in CTC as well as increasing training in the interpretation of CT imaging, and this may contribute to further improvement in the quality of CTCs and its interpretation in the future. ESGE currently recommends CTC for patients unfit for colonoscopy or patients who have had an incomplete colonoscopy. ${ }^{49} \mathrm{How}-$ ever, at present, there is insufficient evidence to support CTC as a first-line investigation in patients with positive $\mathrm{gFOBt} .{ }^{49}$

\section{Patient experience}

Patient experience is an increasingly important marker of care quality and forms part of the UK Government's commitment to health policy and service improvement. ${ }^{57,58} \mathrm{~A}$ recent inquiry into an NHS Trust with high mortality rates described how loss of sight of patients at the center of health care adversely affected clinical quality. ${ }^{59}$ Ensuring positive patient experience is crucial to ensure maximal screening program participation, in addition to ensuring repeat attendance for surveillance procedures. ${ }^{60,61}$ Although the link between patient experience and colonoscopy outcomes has not been fully explored, it is likely that endoscopists who provide a better patient experience are more likely to offer higher quality endoscopy. ${ }^{62}$ At present, patient experience within the BCSP is measured using a nurse/clinician-derived satisfaction questionnaire, meaning that the items assessed have been devised by health care professionals without patient input. A future challenge for the BCSP will be to incorporate truly patient-reported experience measures to enable assessment of patient experience according to patient-derived priorities. ${ }^{63}$

Patient-reported satisfaction of NHS BCSP colonoscopy is generally positive. More than $95 \%$ of patients felt that they understood the risks and benefits of the procedure, and $>98 \%$ of patients felt respected and that their privacy was maintained during the hospital visit. ${ }^{64}$ About $20 \%$ of patients felt that colonoscopy was more uncomfortable than anticipated, and about $5 \%$ of patients asked for the colonoscopy to be stopped. ${ }^{64}$ Around $12.8 \%-16.9 \%$ of patients experienced abdominal pain post colonoscopy. ${ }^{64}$ In contrast, in patients who underwent CTC, $25.7 \%$ felt it was more uncomfortable than expected, as opposed to $21 \%$ of patients who underwent colonoscopy. ${ }^{52}$ There was no significant difference in unexpected discomfort between procedures among patients who underwent colonoscopy following an abnormal CTC. ${ }^{52}$ 
Conversely, a higher proportion of patients found colonoscopy unexpectedly more uncomfortable following incomplete CTC (57\%) than the CTC itself (26.3\%). ${ }^{52}$ Anorectal pain and abdominal pain were most commonly reported, the cause for this being likely multifactorial, including colonoscopy technique and patient-related factors such as previous abdominal surgery and poor patient tolerance.

The difference in perceptions of comfort for the investigation modality may vary due to patient expectations or from lack of counseling prior to either investigation. As CTC may be perceived as more comfortable compared to colonoscopy, this may account for the higher levels of unexpected discomfort for patients receiving CTC first line. Overall, from a patient comfort point of view, CTC has been found to be acceptable and comparable to colonoscopy in terms of comfort; however, further work is required to compare the overall patient experience of each modality.

\section{The future: FIT versus FOB}

gFOBt has been used since the commencement of BCSP. Other screening programs in France, Italy, Spain, and Norway use FIT. There are two different versions of FIT kits; one is qualitative and the other quantitative. The former requires a visual interpretation of results, giving rise to the possibility of inter-observer variation. The latter measures the actual fecal hemoglobin concentration. Different kits are available for these two subtypes, and each varies in performance, and therefore, each kit must be considered individually.

One main difference between gFOBt and FIT is the sampling method and number of stool samples collected. Only one fecal sample collection is required for FIT. Fecal sampling also varies in that the sampling stick is re-inserted into the collector as opposed to smeared onto the test card as in gFOBt. With gFOBt, only stool that has not yet been in the toilet bowl is collected. The sampling method and the collection of only one sample may improve the acceptability of gFOBt for nonresponders. In the UK FIT national screening pilot, uptake in patients randomized to the FIT group was higher than gFOBt (66.4\% vs 59.3\%). ${ }^{65}$ More importantly, the uptake of FIT screening test was doubled in previous nonresponders. ${ }^{65}$ Changing from gFOBt to FIT may improve participation of patients in BCSP. In this trial, uptake of colonoscopy irrespective of the stool test kit was similar. ${ }^{65}$

The main difference between the two kits is the detection level of fecal blood concentration. gFOBt kit can detect fecal blood concentration of $0.6 \mathrm{mg} \mathrm{Hb} / \mathrm{g}$, whereas FIT can detect a much lower concentration of $0.02 \mathrm{mg} \mathrm{Hb} / \mathrm{g}$. This large difference in the detection levels has led many to believe that FIT is superior. gFOBt is not specific for colonic human blood; it can also be positive in response to muscle protein, gastrointestinal irritation due to medication or upper gastrointestinal bleeding, whereas FIT is specific for human colonic bleeding. ${ }^{10}$ The PPV of FIT was greater than that of gFOBt for advanced adenoma (1.73\% vs $0.35 \%)$ and all neoplasia (3.74\% vs $0.76 \%) .{ }^{65}$ A meta-analysis identified that FIT has a sensitivity and specificity of $79 \%$ and $94 \%$, respectively. ${ }^{66}$ Additionally, it detects twice more CRCs and advanced adenomas. ${ }^{65}$ Implementation of FIT has also been shown to reduce $\mathrm{CRC}$ incidence by $10 \%{ }^{67}$

In the FIT screening pilot in England, a quantitative FIT kit was used. Various cutoff levels of FIT and its detection rate of neoplasia were evaluated against gFOBt. Using a cutoff of $0.02 \mathrm{mg} \mathrm{Hb} / \mathrm{g}$, FIT was better at detecting CRC $(0.27 \%$ vs $0.12 \%$ ) and adenoma ( $1.73 \%$ vs $0.35 \%) .{ }^{65}$ Lowering the FIT cutoff value may increase the PPV for CRC and ADR; however, this would need to be balanced against the likely resulting increased demand for colonoscopy which could exceed the capacity of BCSP units. The authors felt that with a FIT cutoff of $0.18 \mathrm{mg} \mathrm{Hb} / \mathrm{g}$, the numbers of colonoscopy generated were comparable to the gFOBt arm, but would increase the yield of CRCs and adenomas. ${ }^{65}$

As of June 2016, it has been announced that FIT kit will be rolled out in 2018 to replace gFOBt nationally in England.

\section{Conclusion}

BCSP has been running in England for the past 11 years, and it has been 4 years since Bowelscope was introduced alongside gFOBt screening. A significant stage shift has been observed. The addition of Bowelscope screening will likely reduce $\mathrm{CRC}$ incidence and improve mortality further. However, uptake of bowel cancer screening remains below that of other screening programs, and work to improve palatability and uptake of screening is required. Changing to FIT may further enhance BCSP in terms of anticipated increased participation and increased yield of $\mathrm{CRC}$ or neoplasia. Areas requiring further research to improve on $\mathrm{CRC}$ outcomes could potentially include interval cancers and uptake in previous nonresponders. As the BCSP database provides a wealth of information for research, this will aid further research into bowel cancer screening. Overall, the future of BCSP with a change to FIT and addition of Bowelscope is likely to see further improvements in CRC morbidity and mortality. 


\section{Disclosure}

Colin J Rees has received research funding from ARC medical, Norgine, Olympus Medical, and Aquilant Endoscopy. He has also received honoraria, consultancy, and travel funding from Norgine, Olympus, and Boston Scientific. The authors report no other conflicts of interest in this work.

\section{References}

1. Cancer Research UK. Bowel cancer statistics. Available from: http:// www.cancerresearchuk.org/health-professional/cancer-statistics/ statistics-by-cancer-type/bowel-cancer. Accessed December 15, 2016.

2. Logan RF, Patnick J, Nickerson C, Coleman L, Rutter MD, von Wagner C; English Bowel Cancer Screening Evaluation Committee. Outcomes of the Bowel Cancer Screening Programme (BCSP) in England after the first 1 million tests. Gut. 2012;61(10):1439-1446.

3. PHE screening blog. We're getting FIT for bowel cancer screening. Available from: https://phescreening.blog.gov.uk/2016/07/25/weregetting-fit-for-bowel-cancer-screening. Accessed December 15, 2016.

4. Hardcastle JD, Chamberlain KO, Robinson MHE, et al. Randomised controlled trial of faecal-occult-blood screening for colorectal cancer. Lancet. 1996;348(9040):1472-1477.

5. Atkin W, Wooldrage K, Parkin DM, et al. Long term effects of once-only flexible sigmoidoscopy screening after 17 years of follow-up: the UK Flexible Sigmoidoscopy Screening randomised controlled trial. Lancet. 2017;389(10076):1299-1311.

6. Kronborg O, Fenger C, Olsen J, Jørgensen OD, Søndergaard O. Randomised study of screening for colorectal cancer with faecal-occultblood test. Lancet. 1996;348(9040):1467-1471.

7. Mandel JS, Bond JH, Church TR, et al. Reducing mortality from colorectal cancer by screening for fecal occult blood. Minnesota Colon Cancer Control Study. N Engl J Med. 1993;328(19):1365-1371.

8. Rees CJ, Bevan R. The National Health Service Bowel Cancer Screening Program: the early years. Expert Rev Gastroenterol Hepatol. 2013;7(5):421-437.

9. Cairns SR, Schofield JH, Steele RJ, et al; on behalf of The British Society of Gastroenterology, and the Association of Coloproctology for Great Britain and Ireland. Guidelines for colorectal cancer screening and surveillance in moderate and high risk groups (update from 2002). Gut. 2010;59:666-690.

10. Geraghty J, Butler P, Seaman H, et al. Optimising faecal occult blood screening: retrospective analysis of NHS Bowel Cancer Screening data to improve the screening algorithm. Br J Cancer. 2014;111:2156-2162.

11. Lee TJ, Clifford GM, Rajasekhar P, et al. High yield of colorectal neoplasia detected by colonoscopy following a positive faecal occult blood test in the NHS Bowel Cancer Screening Programme. $J$ Med Screen. 2011;18(2):82-86.

12. Braun M, Hill J, Kuryba, et al; on behalf of The Association of Coloproctology of Great Britain and Ireland (ACPGI), The Royal College of Surgeons of England (RCS). National Bowel Cancer Audit, Annual Report; 2016. Available from: https://www.acpgbi.org.uk/content/ uploads/2016/07/nati-clin-audi-bowe-canc-2016-rep-v2.pdf. Accessed December 15, 2016.

13. Gill MD, Bramble MG, Rees CJ, Lee TJ, Bradburn DM, Mills SJ. Comparison of screen-detected and interval colorectal cancers in the Bowel Cancer Screening Programme. Br J Cancer. 2012;107(3):417-421.

14. Morris EJA, Whitehouse LE, Farrell T, et al. A retrospective observational study examining the characteristics and outcomes of tumours diagnosed within and without of the English NHS Bowel Cancer Screening Programme. Br J Cancer. 2012;107:757-764.

15. Gill MD, Bramble MG, Hull MA, et al. Screen-detected colorectal cancers are associated with an improved outcome compared with stage-matched interval cancers. Br J Cancer. 2014;111:2076-2081.

16. Muto T, Bussey HJR, Morson BC. The evolution of cancer of the colon and rectum. Cancer. 1975;36:2251-2270.
17. Atkin WS, Cuzick J, Northover JM, Whynes DK. Prevention of colorectal cancer by once-only sigmoidoscopy. Lancet. 1993;341(8847):736-740.

18. Segnan N, Senore C, Andreoni B, et al; SCORE Working Group - Italy. Baseline findings of the Italian multicentre randomised controlled trial of "once-only sigmoidoscopy". J Natl Cancer Inst. 2002;94: $1763-1772$.

19. Weissfeld J, Schoen R, Pinsky P, et al; PLCO Project Team. Flexible sigmoidoscopy in the PLCO cancer screening trial: results from the baseline screening examination of a randomized trial. $J$ Natl Cancer Inst. 2005;97:989-997.

20. Holme O, Loberg M, Kalager M, et al. Effect of flexible sigmoidoscopy screening on colorectal cancer incidence and mortality: a randomized clinical trial. JAMA. 2014;312(6):606-615.

21. Atkin WS, Edwards R, Ines K-H, et al; UK Flexible Sigmoidoscopy Trial Investigators. Once-only flexible sigmoidoscopy screening in prevention of colorectal cancer: a multicentre randomised controlled trial. Lancet. 2010;375:1624-1633.

22. Cancer Research UK. Breast cancer diagnosis and treatment statistics. Available from: http://www.cancerresearchuk.org/health-professional/ cancer-statistics/statistics-by-cancer-type/breast-cancer/diagnosis-andtreatment. Accessed May 22, 2017.

23. Cancer Research UK. Cervical cancer diagnosis and treatment statistics. Available from: http://www.cancerresearchuk.org/health-professional/ cancer-statistics/statistics-by-cancer-type/cervical-cancer/diagnosisand-treatment\#heading-Four. Accessed May 22, 2017.

24. UK Colorectal Cancer Screening Pilot Group. Results of the first round of demonstration pilot of screening for colorectal cancer in the United Kingdom. BMJ. 2004;329(7458):133.

25. Cancer Research UK. Bowel Cancer Screening Coverage and Uptake. UK, FY2012-2015. Available from: http://www.cancerresearchuk.org/ sites/default/files/cstream-node/screen_bowel_cov_upt.pdf. Accessed October 21, 2017.

26. Hall NJ, Rubin GP, Dobson C, et al. Attitudes and beliefs of nonparticipants in a population-based screening programme for colorectal cancer. Health Expect. 2015;18(5):1645-1657.

27. Kobayashi LC, Waller J, von Wagner C, Wardle J. A lack of information engagement among colorectal cancer screening non-attenders: crosssectional survey. BMC Public Health. 2016;16:659.

28. Wardle J, von Wagner C, Kralj-Hans I, et al. Effects of evidence-based strategies to reduce the socioeconomic gradient of uptake in the English NHS Bowel Cancer Screening Programme (ASCEND): four clusterrandomised controlled trials. Lancet. 2016;387(10020):751-759.

29. Hirst Y, Skrobanski H, Kerrison RS, et al. Text-message Reminders in Colorectal Cancer Screening (TRICCS): a randomised controlled trial. Br J Cancer. 2017 Epub Apr 25.

30. Brotherstone H, Vance M, Edwards R, et al. Uptake of population-based flexible sigmoidoscopy screening for colorectal cancer: a nurse-led feasibility study. J Med Screen. 2007;14(2):76-80.

31. Robb K, Power E, Kralj-Hans I, et al. Flexible sigmoidoscopy screening for colorectal cancer: uptake in a population-based pilot programme. J Med Screen. 2010;17(2):75-78.

32. McGregor LM, Bonello B, Kerrison RS, et al. Uptake of Bowel Scope (Flexible Sigmoidoscopy) Screening in the English National Programme: the first 14 months. J Med Screen. 2016;23(2):77-82.

33. Hall N, Birt L, Rees CJ, et al. Concerns, perceived need and competing priorities: a qualitative exploration of decision-making and nonparticipation in a population-based flexible sigmoidoscopy screening programme to prevent colorectal cancer. BMJ Open. 2016;6:e012304.

34. Kerrison RS, McGregor LM, Marshall S, et al. Use of a 12 months' self-referral reminder to facilitate uptake of bowel scope (flexible sigmoidoscopy) screening in previous non-responders: a London-based feasibility study. Br J Cancer. 2016;114(7):751-758.

35. McGregor LM, Skrobanski H, Miller H, et al. Using Specialist Screening Practitioners (SSPs) to increase uptake of the Bowel Scope (Flexible Sigmoidoscopy) Screening Programme: a study protocol for a feasibility single-stage phase II trial. Pilot Feasibility Studies. 2016;2:54. 
36. Lee TJW, Rutter MD, Blanks RG, et al. Colonoscopy quality measures: experience from the NHS Bowel Cancer Screening Programme. Gut. 2012;61:1050-1057.

37. Rees CJ, Thomas Gibson S, Rutter MD, et al; on behalf of: the British Society of Gastroenterology, the Joint Advisory Group on GI Endoscopy, the Association of Coloproctology of Great Britain and Ireland. UK key performance indicators and quality assurance standards for colonoscopy. Gut. 2016;65(12):1923-1929.

38. Rees C, Bevan R, Zimmermann-Fraedrich K, et al. Expert opinions and scientific evidence for colonoscopy key performance indicators. Gut. 2016;65(12):2045-2060.

39. Kaminski MF, Regula J, Krasewska E, et al. Quality indicators for colonoscopy and the risk of interval cancer. NEngl JMed. 2010;362:1795-1803.

40. NHS BCSP Publication No 6. Quality assurance Guidelines for colonoscopy. 2011. Available from: https:/www.gov.uk/government/uploads/ system/uploads/attachment_data/file/427591/nhsbcsp06.pdf. Accessed December 15, 2016.

41. Rajasekhar PT, Clifford GM, Lee TJW, et al. Bowel cancer screening is safe, detects earlier stage cancer and adenomas in $50 \%$ of cases: experience of the prevalent round of screening from two first wave centres in the North East of England. Frontline Gastroenterol. 2012;3:10-15.

42. Lee TJ, Rees CJ, Blanks RG, et al. Colonoscopic factors associated with adenoma detection in a national colorectal cancer screening program. Endoscopy. 2014;46(3):203-211.

43. Lee TJ, Blanks RG, Rees CJ, et al. Longer mean colonoscopy withdrawal time is associated with increased adenoma detection: evidence from the Bowel Cancer Screening Programme in England. Endoscopy. 2013;45(1):20-26.

44. Rondonotti E, Zolk O, Amato A, et al. The impact of hyoscine- $N$-butylbromide on adenoma detection during colonoscopy: meta-analysis of randomized, controlled studies. Gastrointest Endosc. 2014;80(6):1103-1112.

45. British Society of Gastroenterology. Safety and sedation during endoscopic procedures; 2003. Available from: http:/www.bsg.org.uk/ pdf_word_docs/sedation.pdf. Accessed December 15, 2016.

46. Ball AJ, Rees CJ, Corfe BM, Riley SA. Sedation practice and comfort during colonoscopy: lessons learnt from a national screening programme. Eur J Gastroenterol Hepatol. 2015;27(6):741-746.

47. Atkin W, Rogers $\mathrm{P}$, Cardwell $\mathrm{C}$, et al. Wide variation in adenoma detection rates at screening flexible sigmoidoscopy. Gastroenterology. 2004;126:1247-1256.

48. Bevan R, Blanks R, Nickerson C, et al. PWE-365 Exploring ADR performance in the NHS bowel scope programme. Gut. 2015;64:A371.

49. Spada C, Stoker J, Alarcon O, et al. Clinical indications for computed tomographic colonography: European Society of Gastrointestinal Endoscopy (ESGE) and European Society of Gastrointestinal and Abdominal Radiology (ESGAR) Guideline. Endoscopy. 2014;46:897-908.

50. Pickhardt PJ, Hassan C, Halligan S, Marmo R. Colorectal cancer: CT colonography and colonoscopy for detection - systematic review and meta-analysis. Radiology. 2011;259(2):393-405.

51. Atkin W, Dadswell E, Wooldrage K, et al. Computed tomographic colonography versus colonoscopy for investigation of patients with symptoms suggestive of colorectal cancer (SIGGAR): a multicentre randomised trial. Lancet. 2013;381(9873):1194-1202.
52. Plumb AA, Ghanouni A, Rees CJ, et al. Patient experience of CT colonography and colonoscopy after fecal occult blood test in a national screening programme. Eur Radiol. 2017;27(3):1052-1063.

53. Bellini D, Rengo M, De Cecco CN, et al. Perforation rate in CT colonography: a systematic review of the literature and meta-analysis. Eur Radiol. 2014;24(7):1487-1496.

54. Perisinakis K, Seimenis I, Tzedakis A, et al. Screening computed tomography colonography with 256-slice scanning: should patient radiation burden and associated cancer risk constitute a major concern? Invest Radiol. 2012;47(8):451-456.

55. Plumb AA, Halligan S, Nickerson C, et al. Use of CT colonography in the English Bowel Cancer Screening Programme. Gut. 2013;63(6): 964-973.

56. Plumb AA, Halligan S, Pendsé DA. Sensitivity and specificity of CT colonography for the detection of colonic neoplasia after positive faecal occult blood testing: systematic review and meta-analysis. Eur Radiol. 2014;24(5):1049-1058.

57. Sizmur S, Redding D. Core Domains for Measuring Inpatients'Experience of Care. Oxford: Picker Institute Europe; 2009. Available from http://www.pickereurope.org/assets/content/pdf/Survey_data_analyses/ Discussion_Paper_1_core_domains_inpatient_experience_Dec_09_ final.pdf. Accessed May 22, 2017.

58. Patient Experience. Patient reported outcome measures: their role in measuring and improving patient experience; 2012. Available from: http://patientexperienceportal.org/article/patient-reportedoutcomemeasurestheir-role-in-measuring-and-improving-patient-experience. Accessed May 22, 2017.

59. Francis R. Report of the Mid Staffordshire NHS Foundation Trust Public Inquiry. London: Stationery Office HC 947; 2013.

60. Black N, Jenkinson C. How can patients' views of their care enhance quality improvement? BMJ. 2009;339:202-205.

61. Hydes T, Hansi N, Trebble TM. Lean thinking transformation of the unsedated upper gastrointestinal endoscopy pathway improves efficiency and is associated with high levels of patient satisfaction. BMJ Qual Saf. 2012;21:63-69.

62. Ekkelenkamp VE, Shaw I, Valori R, Dunckley P. Comfort scores in colonoscopy performance. Gut. 2011;60:A44-A45.

63. Brown S, Bevan R, Rubin G, et al. Patient-derived measures of GI Endoscopy: a meta-narrative review of the literature. Gastrointest Endosc. 2015;81(5):1130-1140.

64. Ghanouni A, Plumb A, Hewitson P, Nickerson C, Rees CJ, von Wagner C. Patients' experience of colonoscopy in the English Bowel Cancer Screening Programme. Endoscopy. 2016;48:232-240.

65. Moss S, Mathews C, Day TJ, et al. Increased uptake and improved outcomes of bowel cancer screening with a faecal immunochemical test: results from a pilot study within the national screening programme in England. Gut. 2016;66(9):1631-1644.

66. Lee JK, Liles EG, Bent S, Levin TR, Corley DA. Accuracy of fecal immunochemical tests for colorectal cancer: systematic review and meta-analysis. Ann Intern Med. 2014;160:171.

67. Giorgi Rossi P, Vicentini M, Sacchettini C, et al. Impact of screening program on incidence of colorectal cancer: a cohort study in Italy. Am J Gastroenterol. 2015;110(9):1359-1366.
Risk Management and Healthcare Policy

\section{Publish your work in this journal}

Risk Management and Healthcare Policy is an international, peer-reviewed open access journal focusing on all aspects of public health, policy, and preventative measures to promote good health and improve morbidity and mortality in the population. The journal welcomes submitted papers covering original research, basic science, clinical and epidemiological studies, reviews and evaluations, guidelines, expert opinion and commentary, case reports and extended reports. The manuscript management system is completely online and includes a very quick and fair peerreview system, which is all easy to use. Visit http://www.dovepress.com/ testimonials.php to read real quotes from published authors. 